Service social

Les mobilisations populaires urbaines, par Pierre Hamel, Jean-François Léonard et Robert Mayer, Montréal, Nouvelle Optique, 1982, 482 pages

L'intervention communautaire, par Henri Lamoureux, Robert Mayer et Jean Panet-Raymond, Montréal, Éditions Saint-Martin, 1984, 237 pages.

\title{
Jean-Pierre Deslauriers
}

Volume 34, numéro 2-3, 1985

L'organisation communautaire

URI : https://id.erudit.org/iderudit/706287ar

DOI : https://doi.org/10.7202/706287ar

Aller au sommaire du numéro

Éditeur(s)

École de service social de l'Université Laval

ISSN

1708-1734 (numérique)

Découvrir la revue

Citer ce compte rendu

Deslauriers, J.-P. (1985). Compte rendu de [Les mobilisations populaires urbaines, par Pierre Hamel, Jean-François Léonard et Robert Mayer, Montréal, Nouvelle Optique, 1982, 482 pages / L'intervention communautaire, par Henri

Lamoureux, Robert Mayer et Jean Panet-Raymond, Montréal, Éditions

Saint-Martin, 1984, 237 pages.] Service social, 34(2-3), 415-417.

https://doi.org/10.7202/706287ar d'utilisation que vous pouvez consulter en ligne. 
prometteur. J'ai vraiment aimé cette lecture. Si quelqu'un s'avise un jour de publier un corpus scientifique sur les groupes populaires, Deslauriers et Pouliot devront y prendre place, non pas en raison de l'originalité de leur sujet ou de leur méthodologie, mais bien pour la qualité du traitement, la rigueur de la méthode et l'adresse du style.

Travailleurs sociaux et cadres institutionnels qui n'affichez plus l'assurance d'autrefois devant la sacro-sainte efficacité organisationnelle, cette lecture pourrait éclairer votre lanterne en ouvrant la voie à une alternative plausible. Combien de temps encore nous faudra-t-il bûcher en vain et marquer le pas avant qu'on ne se ressaisisse de cette mégalomanie organisationnelle?

École de service social,

Laval DOUCET

Université Laval.

Les mobilisations populaires urbaines, par Pierre HAMEL, Jean-François LÉONARD et Robert MAYer, Montréal, Nouvelle Optique, 1982, 482 pages ; L'intervention communautaire, par Henri LAMOUREUX, Robert MAYER et Jean PANETRAYMOND, Montréal, Éditions Saint-Martin, 1984, 237 pages.

Écrits à quelques années d'intervalle, ces deux livres se complètent. Tous deux poursuivent le même but : relever les traces, dépasser la tradition orale, apprendre de l'expérience passée. Le premier présente une brochette de textes rendant compte des temps forts des groupes populaires depuis une vingtaine d'années. Comme le disent les auteurs: "Nous avons voulu, par ce moyen [le recueil de textes], faciliter l'accès à des documents significatifs, à des témoignages de première main, à des récits de luttes oubliées et la plupart du temps considérées comme marginales et officiellement marginalisées par les médias. Faciliter l'accès à des documents pour raviver la mémoire collective. Entreprise importante si l'on considère que plusieurs de ces matériaux de base ont été dispersés aux quatre vents, au gré des aventures collectives et qu'il est parfois difficile d'y avoir accès." (P. 8.)

Le livre est divisé en quatre parties. La première traite de l'évolution des groupes populaires et met l'accent sur les débats, événements et batailles qui ont marqué les efforts des personnes pour améliorer leurs conditions de vie. La deuxième porte sur le rôle des intellectuels dans le mouvement populaire et, comme soulignent les auteurs: "ll est relié à l'hypothèse d'une tendance à l'homogénéisation des conditions de vie dans le capitalisme avancé pour l'ensemble des classes populaires, y compris la nouvelle petite bourgeoisie" (p. 12). Hypothèse intéressante qui relancerait la discussion sur une autre piste que celle de l'avant-garde pourvu que l'on se débarrasse de l'obsession de la paupérisation qui en ferait un prolétaire malgré lui. Le troisième thème examine les rapports de l'État et des groupes populaires en s'attardant à la Révolution tranquille. Enfin, la dernière partie considère les expériences populaires sur le terrain de l'action politique municipale. Outre l'introduction 
générale, chaque partie est précédée d'une introduction spécifique qui donne le ton et présente les textes. Une bibliographie fort complète suit chaque grand chapitre et s'avérera d'une grande utilité pour ceux et celles qui voudront en savoir davantage. Une conclusion générale manque malheureusement à l'appel.

Ce recueil est volumineux et peut-être aurait-il été préférable de restreindre le nombre de thèmes et de se limiter aux textes inédits : en effet, si les auteurs voulaient publier des documents difficiles d'accès, ils auraient pu supprimer les articles de revues, les publications d'organismes officiels tels que le Conseil de développement social et la Commission d'enquête sur la santé et le bien-être social. De plus, une trop grande diversité rend la cohérence difficile et déroute le lecteur. Ensuite, si les expériences et les documents se rapportent au milieu montréalais, j'aurais aimé que le titre soit moins ambitieux et précise que le volume allait présenter les expériences de mobilisation populaire à Montréal! Quoi qu'il en soit, cet ouvrage constituera un outil précieux pour ceux et celles qui s'intéressent à la question : ce livre compare des points de vue différents et parfois opposés, il est bien documenté, le ton n'est pas dogmatique, et il avance une synthèse originale de l'évolution des groupes populaires.

Alors que cet ouvrage est plus théorique et plus analytique, celui de Lamoureux, Mayer et Panet-Raymond est plus pratique et se présente comme un manuel d'intervention communautaire. Ce volume navigue avec succès entre plusieurs écueils : il évite le romantisme communautaire qui sombre dans une vision trop idéaliste de la réalité ; il contourne l'utilisation d'une méthodologie formelle qui s'en tiendrait à la recette (de ce point de vue, Dunham apparaît d'une souplesse étonnante par rapport aux marxistes-léninistes !) ; il prend garde aussi de confondre l'action communautaire avec la politique pure et simple, même si la politique est toujours présente. Les auteurs tentent plutôt d'épouser les contours de l'action populaire et de développer une méthode qui tienne compte des caractéristiques de l'organisation populaire, telles que la démocratie, la souplesse des structures et l'importance accordée aux personnes.

Ce livre reprend les divisions qu'on retrouve habituellement dans un manuel de ce genre: rappel des lieux de pratique, l'organisation et son fonctionnement, le financement, etc. Cependant, son originalité réside dans la part réservée à la recherche. Trop souvent, on oppose le chercheur-théoriciendébranché à l'organisateur-praticien-connecté. Dans une couple de chapitres, les auteurs démontrent l'utilité et la pertinence de la recherche et de l'analyse en intervention communautaire, et ce n'est pas courant.

Comme la société dans son ensemble, l'intervention communautaire traverse une passe difficile : il est malaisé d'y développer une perspective à long terme, on remet en question le statut de l'animateur, de l'organisateur, de l'intervenant, de l'intellectuel; la vie politique se redéfinit par rapport à la vie privée. Toutefois, en dépit des changements sociaux et économiques, les personnes continuent de vivre dans des réseaux, dans des communautés qui peuvent être réactivés et se transformer en force de changement, le moment venu. "Le mouvement populaire fut et est encore un des lieux privilégiés où s'expriment la sagesse et la ténacité de notre peuple. Loin d'être épuisé, le 
dynamisme de ce mouvement pourrait bien être une source de changements encore plus importants et surtout, plus significatifs." (P. 18.) On ne saurait mieux dire.

Département des sciences humaines,

Jean-Pierre DESLAURIERS

Université du Québec à Chicoutimi.

Faut-il brûler les pancartes? Le mouvement populaire aujourd'hui, par Jean Panet-Raymond et Jean-François René, Montréal, Éditions ACEF du Centre de Montréal, 1984, 94 pages.

Dans sa conception, l'ouvrage se veut le reflet de l'engagement prolongé de deux militants de l'Association coopérative d'économie familiale (ACEF) aujourd'hui liés au secteur de l'organisation communautaire de l'École de service social de l'Université de Montréal. Sa pertinence, sa signification et sa cohérence reposent en bonne partie sur cet engagement réel et sur le fait que les auteurs ont voulu faire un ouvrage en collaboration avec des gens qui font I'histoire des groupes populaires. Ils qualifient leur ouvrage de "fruit d'un dialogue et d'un échange" entre des intellectuels et des militants et des militantes qui ont vécu ou vivent encore l'histoire des groupes étudiés.

Ils ont choisi trois groupes populaires qui ont écrit des pages importantes du mouvement ouvrier et populaire de Montréal: l'ACEF de Montréal, Le Regroupement pour le gel des loyers et l'Association pour la défense des droits sociaux. Pour réaliser cette monographie critique, ils ont suivi un processus de recherche-action en liant deux méthodes différentes: l'étude de documents et les entrevues ouvertes avec des témoins de l'action étudiée. Le moins que l'on puisse dire c'est que la démarche suivie fut dynamique et riche.

Le regard sur l'histoire de professionnels engagés est rarement terne. Panet-Raymond et René ne font pas exception et situent l'origine de leur ouvrage dans le refus de se laisser enfermer dans un processus irréversible de I'histoire. Ils croient fermement que celle-ci peut éclairer l'avenir, c'est pourquoi ils tentent de démontrer comment les groupes populaires étudiés traduisent en microcosme les efforts de changement socio-politique de la nation québécoise. Ainsi ils fournissent des éléments de réponse aux profondes interrogations ou remises en question que vivent les organisations populaires depuis quelques années. Leur effort en ce sens contribue à faire mieux comprendre les limites et les forces des organisations populaires. La position des auteurs est donc claire et elle sort le lecteur ou la lectrice d'un regard froid et statique sur une réalité sociale. Le lien entre l'analyse théorique et la pratique est réel et se traduit par un discours dynamique.

Dans leur interrogation des pratiques, les auteurs ont privilégié trois dimensions fondamentales: le discours véhiculé par les organisations, leur fonctionnement démocratique et leur orientation politique. Tout au long de l'analyse, ils dégagent clairement l'évolution de chaque organisation en identifiant des phases précises. À l'intérieur de chaque phase, il est facile de 